DOI: https://dx.doi.org/10.26808/rs.ca.i9v6.01 International Journal of Computer Application (2250-1797)

Issue 9 Volume 6, November-December 2019

\title{
A Survey on Hyperspectral Image Classification using Adaptive Spatial-Spectral Feature Learning
}

\author{
1 G.Elayaroja, ${ }^{2}$ V. Uma Sankari \\ ${ }^{1}$ Assistant Professor, Department of Computer Science and Engineering, \\ CK College of Engineering and Technology, Cuddalore. \\ e-mail : rojagovindan@gmail.com \\ ${ }^{2}$ Assistant Professor, Department of Computer Science and Engineering, \\ CK College of Engineering and Technology, Cuddalore. \\ e-mail : umavijayakumar04@gmail.com
}

\begin{abstract}
The combination of spectral and spatial information provides an effective way to improve the hyperspectral image (HSI) classification. Hyperspectral image processing has been a very dynamic area in remote sensing and other applications in recent years. Traditional methods, have shown promising results in hyperspectral image classification. Such methodologies, nevertheless, can lead to information loss in representing hyperspectral pixels, which intrinsically have a sequence-based data structure. In the implementation of the proposed method, a convolution neural network (CNN) is first applied to learn weight features for each pixel within a hyperspectral patch and adaptive weights can be obtained based on a softmax normalization. Then, the shallow joint adaptive features can be acquired according to these weights. After that, a stacked auto-encoder (SAE) is proposed to further extract deeper hierarchical features for the final classification.
\end{abstract}

Keywords- Adaptive spatial-spectral features, convolutional neural network (CNN), hyperspectral image (HSI) classification, stacked auto-encoder (SAE).

\section{INTRODUCTION}

Remote sensing can be defined as collection and interpretation of information about an object, area or event without any physical contact with the object. Aircraft and satellites are the common platforms for remote sensing of earth and its natural resources (Goetz et al., 1985). Digital image processing is a process where input image is processed to get output also as an image or attributes of the image. Main aim of all image processing techniques is to recognize the image or object under consideration easier visually. It is used of extracting some useful information from original image. It is collects and processes information from across the electromagnetic spectrum. The goal of hyperspectral imaging is to obtain the spectrum for each pixel in the image of a scene, with the purpose of finding objects, identifying materials, or detecting processes.

Image Calibration Image calibration also referred to as camera resectioning, estimates the parameters of a lens and image sensor of an image or video camera. Image Segmentation Image segmentation is the separation of an picture into regions or categories, which address to separate objects or parts of objects.

Image Extraction Image processing, form extraction begin from an opening set of moderated data and builds deduce values (features) intended to be instructive and nonredundant, facilitating the succeeding learning and generalization measure. 
DOI: https://dx.doi.org/10.26808/rs.ca.i9v6.01 International Journal of Computer Application (2250-1797)

Issue 9 Volume 6, November-December 2019

Multivariate Data Analysis It is refers to any statistical technique used to analyze data that arises from more than one variable. This essentially pattern loyalty where each condition, product, or division involves more than a individual variable.

Classification Model A classification technique (or

classifier) is a methodical approach to construction classification design from an input data set. Examples contain decision tree classifiers, regulation-based classifiers, neural networks, support vector machine etc..,

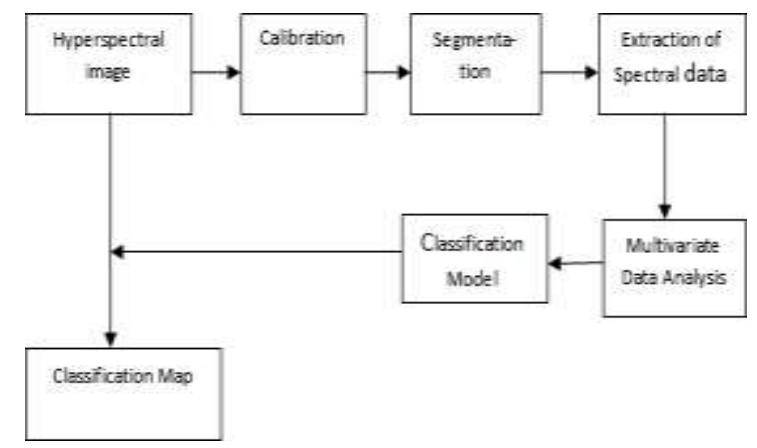

Fig1. Steps Involved In Hyperspectral Image Classification

\section{METHODS FOR HYPERSPECTRAL IMAGE CLASSIFICATION}

\section{A. Recurrent Neural Network (RNN)}

An RNN is a class of artificial neural network that extends the conventional feedforward neural network with loops in connections. Unlike a feedforward neural network, an RNN is able to process the sequential inputs by having a recurrent hidden state whose activation at each step depends on that of the previous step. In this manner, the network can exhibit dynamic temporal behavior. The idea behind RNNs is to make use of sequential information. In a old-fashioned neural network we appropriate that all inputs (and outputs) are independent of each other. But for many work that's a very bade idea If you want to predict the next term in a significance you better know which words came before it. RNNs are called recurrent for they finish the same work for every constitute of a result, with the product being depended on the former computations.

$\mathbf{h}_{\mathrm{t}}=\boldsymbol{\omega}\left(\mathbf{W} \mathbf{x}_{\mathbf{t}}+\mathbf{U h} \mathbf{h}_{\mathrm{t}-1}\right)$

\section{B. Long Short-Term Memory Units (Lstms)}

LSTMs help sustain the failure that can be back propagated through period and layers By continue a more constant failure, they assign recurrent nets to continue to study over many period steps (over 1000), thereby beginning a channel to link causes and result remotely. This is one of the central challenges to machine learning and AI, since algorithms are frequently confronted by environments where reward signals are sparse and delayed, such as life itself LSTMs hold information external the regular flow of the recurrent network in a gated cell. Information can be stored in, written to, or explain from a cell, much similar data in a computer's memory. The cell makes decisions about what to store and when to allow reads, writes and erasures, via gates that open and close. Unlike the digital warehousing on computers, however, these gates are analog, accomplish with element-wise augmentation by sigmoids, which are all in the rank of 01. Analog has the benefit over digital of being differentiable, and therefore becoming for backpropagation. 


\section{Gated Recurrent nit (GRU)}

A gated recurrent unit (GRU) is basically an LSTM without an output gate, which therefore fully writes the contents from its memory cell to the larger net at each time step. A GRU has two gates, a replace gate, and an update gate . Intuitively, the replace gate limit how to combine the modern input with the prior memory, and the update gate explain how much of the prior memory to keep around. If set the reset to all 1 's and update gate to all 0's again arrive at our plain RNN model. The basic idea of using a gating mechanism to learn long-term dependencies is the same as in a LSTM.

\section{RELATED WORKS}

Farid Melgani and Lorenzo Bruzzone [1] proposed a novel SVMs used to solve multiclass problems in hyperspectral data. This paper addresses the problem of the classification of hyperspectral remote sensing images by support vector machines (SVMs). First, a theoretical disputation and trial analysis scope at perception and assessing the potentialities of SVM class ifiers in hyper dimensional feature spaces. Then, assess the effectiveness of SVMs with respect to conventional feature-reductionbased approaches and their performances in hyper subspaces of various dimensionalities. SVMs are a sufficient and powerful disjunctive to formal pattern recognition approaches (feature-reduction procedures combined with a classification process) for the classification of hyperspectral remote sensing data.

JiSoo Ham, Yangchi Chen, Melba M. Crawford and Joydeep Ghosh [2] proposed a novel Random forest used to improved generalization of the classifier in analysis of hyperspectral data. This paper investigates two approaches based on the concept of random forests of classifiers implemented within a binary hierarchical multiclassifier system, with the goal of achieving improved generalization of the classifier in analysis of hyperspectral data, especially when the amount of training data is restricted. A modern classifier is proposed that incorporeal bagging of manege samples and adaptative random subspace form selection within a binary hierarchic classifier (BHC), such that the number of features that is choose at each node of the tree is dependent on the amount of combined training data.

Yushi Chen, Hanlu Jiang, Chunyang Li, Xiuping Jia, and Pedram Ghamisi [3] proposed a novel CNN used to extract effective spectral-spatial features of hyperspectral image. The proposed approximate employs several convolutional and pooling layers to citation deep form from HSIs, which are nonlinear, discriminant, and invariable. These form are useful for image classification and target discernment. More importantly, a 3-D CNN-supported FE model with combined regularization to extract effective spectral-spatial form of hyperspectral images. In summary, to address the HSI FE and classification problem with restricted training example, an judgment of big network with valid constraints. The proposed model can be combined with postclassification processing to enhance mapping performance. It deserves to be investigated as a possible future work.

Haobo Lyu and Hui Lu [4] proposed a novel for Improved LSTM model to learn the binary differencing information for multi-temporal remote sensing data. In this paper, a novel change detection method learned from Recurrent Neural Network with transferable ability is proposed. The proposed method, which is based on an improved Long Short Term Memory (LSTM) model, aims at: 1) learning a novel change detection rule to distinguish changed regions with high accuracy; 2) analyzing a new target data 
DOI: https://dx.doi.org/10.26808/rs.ca.i9v6.01 International Journal of Computer Application (2250-1797)

Issue 9 Volume 6, November-December 2019

with transferable ability from learned change rule; 3) learning the differencing information and detecting the changes independently without any classifiers. In the process of learning the change rule, a core memory cell is utilized to detect and record the differencing information in multi-temporal images.

Adolfo Martinez-Uso Filiberto Pla Jose Martinez Sotoca and Pedro Garcia-Sevilla [5] propose a novel for an approach is used to reduce data redundancy and nonusefulinformation among image bands. The proposed method is based on a hierarchical clustering structure to group bands to minimize the intracluster variance and maximize the intercluster variance. This aim is pursued using information measures, such as distances based on mutual information or Kullback-Leibler divergence, in order to reduce data redundancy and nonuseful information among image bands. Joseph C. Harsanyi and Chein-I Chang [6] propose a novel for an approach for simultaneously reducing hyperspectral data measurement and arrange the hyperspectral image. Most applications of hyperspectral imagery need processing techniques which realize two fundamental goals: 1) detect and classify the constituent materials for each pixel in the scene; 2) reduce the data volume dimensionality, without loss of critical information, so that it can be processed efficiently and assimilated by a human analyst.

Pal, M. and Foody, G. M. [7] proposed a novel for Feature selection is valuable analysis operations for classification by a SVM. SVM are attractive for the classification of remotely sensed data with some claims that the method is insensitive to the dimensionality of the data and so not requiring a dimensionality reduction analysis in pre-processing. Here, a sequence of classification analyses with two hyperspectral sensor data sets reveal that the correctness of a classification by a SVM does modify as a function of the many of features used. Critically, it is shown that the correctness of a classification may decline significantly (at 0.05 level of statistical significance) with the augmentation of features, especially if a small training example is used. This foreground a dependence of the correctness of classification by a SVM on the measurement of the data and so the potentially value of undertaking a feature selection analysis previous to classification.

Yushi Chen, Member, Zhouhan Lin, Xing Zhao, Student Member, Gang Wang, and Yanfeng $\mathrm{Gu},[8]$ propose a novel for Deep learning framework to merge the spectral and spatial information approach for highest classification accuracy. Classification is one of the most familiar topics in hyperspectral remote sensing. In the last two decades, a vast numeral of methods were intend to share with the hyperspectral data classification problem. However, most of them do not hierarchically extract deep features. In this paper, the conception of deep learning is insert into hyperspectral data classification for the first period. A novel deep learning framework to merge the two features, from which get the highest classification accuracy. The framework is a crossbreed of principle component analysis (PCA), deep learning structure, and logical regression. Specifically, as a deep learning architecture, stacked auto encoders are aimed to get useful high-level features.

Suju Rajan, Joydeep 3 Ghosh, and Melba M. Crawford [9] propose a novel for an active learning access for effectively updating classifiers build from trivial quantities of labeled data. an active learning technique that efficiently updates exis ting classifiers by using fewer labeled data points than semi supervised methods. Further, unlike semi supervised methods, our proposed technique is well suited for learning or adapting 
DOI: https://dx.doi.org/10.26808/rs.ca.i9v6.01 International Journal of Computer Application (2250-1797)

Issue 9 Volume 6, November-December 2019

classifiers when there is substantial change in the spectral signatures between labeled and unlabeled data. Thus, our active learning approach is also beneficial for distribute a series of spatially/secularly related images, wherein the spectral signatures modify across the images Our interfoliate semi supervised active learning process was standard on both divide and spatially/temporally related hyperspectral data sets . Present empirical results that establish the superior performance of our proposed approach versus other active learning and semi supervised methods.

Frédéric Ratle, Gustavo Camps-Valls, and Jason Weston [10] proposed a novel for framework for semi- supervised remote sensing image classification based on neural networks is presented. The methodology comprise of adding a flexible embedding regularizer to the damage performance used for training neural networks. Training is done using conjectural gradient descent with supplemental comparison on straints to escape falling into local minimums. The method constitutes a generalization of both supervised and unsupervised methods, and can handle millions of unlabeled samples. Therefore, the proposed approach gives rise to an operational classifier, as opposed to previously presented transductive or Laplacian support vector machinesThe intend methodology compose a common framework for construction computationally effectual semi-supervised methods. The procedure is compare with Lap SVM and TSVM in semisupervised scenarios, to SVM in supervised settings, and to online and batch k-means for unsupervised learning. Results demonstrate the improved classification accuracy and scalability of this approach on several hyperspectral image classification problems

Table -1: Summarization of literature survey

\begin{tabular}{|c|c|c|c|c|}
\hline Sl. NO & TITLE & METHODS & ADVANTAGE & DISADVANTAGE \\
\hline 1 & \begin{tabular}{|c|} 
Classification of \\
Hyperspectral \\
Remote Sensing \\
Images with \\
Support Vector \\
Machines \\
\end{tabular} & $\begin{array}{l}\text { Feature-reduction } \\
\text { Pattern recognition }\end{array}$ & \begin{tabular}{|c|} 
More classification \\
accuracy, Computational \\
time, Stability to \\
parameter setting.
\end{tabular} & $\begin{array}{l}\text { SVM gives some information } \\
\text { loss in hyperspectral data. }\end{array}$ \\
\hline 2 & \begin{tabular}{|c|} 
Investigation of \\
the Random Forest \\
Framework for \\
Classification of \\
Hyperspectral \\
Data.
\end{tabular} & \begin{tabular}{|} 
Binary hierarchical \\
classifier $(\mathrm{BHC})$ \\
classification and \\
regression trees \\
$(\mathrm{CART})$
\end{tabular} & $\begin{array}{c}\text { Improving generalization } \\
\text { Greater Diversity higher } \\
\text { accuracies }\end{array}$ & $\begin{array}{c}\text { Random forest gives some } \\
\text { Data loss in hyperspectral } \\
\text { data. }\end{array}$ \\
\hline 3 & $\begin{array}{c}\text { Deep Feature } \\
\text { Extraction and } \\
\text { Classification of } \\
\text { Hyperspectral } \\
\text { Images Based on } \\
\text { Convolutional } \\
\text { Neural Networks }\end{array}$ & $\begin{array}{l}\text { Feature extraction } \\
\text { (FE) }\end{array}$ & $\begin{array}{c}\text { CNN can extract the } \\
\text { spectral-spatial features } \\
\text { effectively, High } \\
\text { classification accuracy }\end{array}$ & $\begin{array}{l}\text { Limited training samples, } \\
\text { Problem of overfitting. }\end{array}$ \\
\hline
\end{tabular}


DOI: https://dx.doi.org/10.26808/rs.ca.i9v6.01

International Journal of Computer Application (2250-1797)

Issue 9 Volume 6, November-December 2019

\begin{tabular}{|c|c|c|c|c|}
\hline 4 & $\begin{array}{c}\text { Learning A } \\
\text { Transferable } \\
\text { Change Detection } \\
\text { Method by } \\
\text { Recurrent Neural } \\
\text { Network }\end{array}$ & $\begin{array}{l}\text { Multi-temporal } \\
\text { images. }\end{array}$ & $\begin{array}{l}\text { Higher accuracy for } \\
\text { change detection, effective } \\
\text { transferable ability. }\end{array}$ & Less training time. \\
\hline 5 & $\begin{array}{c}\text { Clustering-Based } \\
\text { Hyperspectral } \\
\text { Band Selection } \\
\text { Using Information } \\
\text { Measures }\end{array}$ & $\begin{array}{c}\text { Hierarchical clustering } \\
\text { Kullback-Leibler } \\
\text { divergence }\end{array}$ & $\begin{array}{l}\text { Performance increases in } \\
\text { image classification } \\
\text { tasks. Higher accuracy }\end{array}$ & $\begin{array}{l}\text { Band dissimilarity space } \\
\text { plays in the problem of } \\
\text { classification. }\end{array}$ \\
\hline 6 & $\begin{array}{l}\text { Hyperspectral } \\
\text { Image } \\
\text { Classification and } \\
\text { Dimensionality } \\
\text { Reduction: An } \\
\text { Orthogonal } \\
\text { Subspace } \\
\text { Projection } \\
\text { Approach }\end{array}$ & $\begin{array}{l}\text { Minimum distance and } \\
\text { Maximum likelihood } \\
\text { classifiers Principal } \\
\text { components } \\
\text { analysis(PCA) }\end{array}$ & $\begin{array}{c}\text { To solve a particular } \\
\text { Detection and } \\
\text { classification problem. } \\
\text { Higher accuracy }\end{array}$ & $\begin{array}{l}\text { Suffer from the mixed pixel } \\
\text { problem. }\end{array}$ \\
\hline 7 & $\begin{array}{c}\text { Feature selection } \\
\text { for classification of } \\
\text { hyperspectral data } \\
\text { by SVM }\end{array}$ & Feature selection & $\begin{array}{c}\text { High potential rate. } \\
\text { Higher classification } \\
\text { accuracy. } \\
\text { Computationally efficient }\end{array}$ & $\begin{array}{l}\text { One problem often noted } \\
\text { in the classification of } \\
\text { hyperspectral data is the } \\
\text { Hughes effect or } \\
\text { phenomenon }\end{array}$ \\
\hline 8 & $\begin{array}{c}\text { Deep Learning- } \\
\text { Based } \\
\text { Classification of } \\
\text { Hyperspectral } \\
\text { Data }\end{array}$ & $\begin{array}{c}\text { Stacked } \\
\text { autoencoder(SAE) } \\
\text { Logistic regression } \\
\text { Support vector machine } \\
\text { (SVM). }\end{array}$ & $\begin{array}{l}\text { Highest accuracy when } \\
\text { compared with other } \\
\text { feature extraction } \\
\text { methods.Both SAE-LR } \\
\text { and SVM have proved } \\
\text { the effective result }\end{array}$ & $\begin{array}{l}\text { Testing time efficiency is } \\
\text { less. Less training time. }\end{array}$ \\
\hline 9 & \begin{tabular}{|c} 
An Active \\
Learning \\
Approach to \\
Hyperspectral \\
Data Classification
\end{tabular} & $\begin{array}{l}\text { Semi supervised } \\
\text { learning Active } \\
\text { learning }\end{array}$ & \begin{tabular}{|c|} 
Using very few labeled \\
data points.Provide a high \\
performance. Better \\
learning rates.
\end{tabular} & $\begin{array}{l}\text { Real life problems have large } \\
\text { amount of unlabeled data. }\end{array}$ \\
\hline 10 & $\begin{array}{c}\text { Semi-Supervised } \\
\text { Neural Networks } \\
\text { for Efficient } \\
\text { Hyperspectral } \\
\text { Image } \\
\text { Classification }\end{array}$ & $\begin{array}{c}\text { Transductive SVM, } \\
\text { and Laplacian SVM }\end{array}$ & $\begin{array}{c}\text { Higher accuracy, Higher } \\
\text { performance }\end{array}$ & $\begin{array}{c}\text { Computationally more } \\
\text { expensive }\end{array}$ \\
\hline
\end{tabular}

\section{CONCLUSION}

In this survey, a novel HSI classification framework, namely adaptive spectral-spatial feature learning network (ASSFL), to extract both spatial and spectral infor- mation adaptively within a unified structure. Compared with the traditional classification methods, 
DOI: https://dx.doi.org/10.26808/rs.ca.i9v6.01 International Journal of Computer Application (2250-1797)

Issue 9 Volume 6, November-December 2019

our proposed model considers different local spatial contexture of input hyper- spectral patches during spatial feature integration and intro- duces a weight learning network which consists of a CNN and a softmax normalization to generate adaptive weights given different hyperspectral patches. This could reduce the possi- bility of bringing in inappropriate spatial information, such as noise contaminated samples or samples from other classes. The shallow joint adaptive features are then obtained based on these generated weights and fed to a SAE for higher-level joint spatial-spectral feature learning. Benchmark results on four public HSI data sets demonstrate that our ASSFL has the superior performance with the cleanest classification map and the highest $O A$ values out of other baseline methods. The visualizations of adaptive weights and noise robustness experiments demonstrate the effectiveness of the adaptive feature learning in integrating robust and discriminative joint spatial-spectral features from HSI data sets. Since the adap- tive joint feature learning technique has been proven to be effective in the HSI classification, we shall further explore the characteristics of weight learning network with some supervised constraints in our future work.

\section{REFERENCES}

[1] Lichao Mou, Student Member IEEE, Pedram Ghamisi, Member IEEE, And Xiao Xiang Zhu, Senior Member IEEE, "Deep Recurrent Neural Networks For Hyperspectral Image Classification" IEEE Transactions On Geoscience And Remote Sensing, Vol. 55, No. 7, July 2017.

[2] F. Melgani And L. Bruzzone, "Classification Of Hyperspectral Remote Sensing Images With Support Vector Machines", IEEE Transactions Geo science And Remote Sensing, Vol. 42, No. 8, pp. 1778- 1790, Aug 2004.

[3] J. Ham, Y. Chen, M. M. Crawford, and J. Ghosh, "Investigation of the random forest framework for classification of hyperspectral data", IEEE Transaction Geoscience And Remote Sensing, Vol. 43, No. 3, pp. 492-501, Mar 2005.

[4] Y. Chen, H. Jiang, C. Li, X. Jia, and P. Ghamisi, "Deep feature extraction and classification of hyperspectral images based on convolutional neural networks", IEEE Transaction Geoscience And Remote Sensing, Vol. 54, No. 10, pp. 62326251, Oct 2016.

[5] H. Lyn, H. Lu, and L. Mou, "Learning a transferable change rule from a recurrent neural network for land cover change detection", Remote Sensing, Vol. 8, No. 6, pp. 506, 2016.

[6] Adolfo Martinez-Uso, Filiberto Pla, Jose Martinez Sotoca, and Pedro GarciaServilla, "Clustering- Based Hyperspectral Band Selection Using Information Measures", IEEE Transactions on Geoscience And Remote Sensing, Vol. 45, No. 12, December 2007.

[7] Joseph C. Harsanyi, Member IEEE, and Chein-I Chang, Senior Member IEEE, “ Hyperspectral Image Classification and Dimensionality Reduction: An Orthogonal Subspace Projection Approach", IEEE Transactions on Geoscience And Remote Sensing, Vol. 32, No. 4, July 1994.

[8] Pal, M. and Foody, G. M., "Feature selection for classification of hyperspectral data by SVM", IEEE Transactions on Geoscience And Remote Sensing, Vol. 48, pp. 2297-2307, 2010.

[9] Yushi Chen, Member IEEE, Zhouhan Lin, Xing Zhao, Student Member IEEE, Gang Wang, Member IEEE, and Yanfeng Gu, Member IEEE, "Deep LearningBased Classification of Hyperspectral Data", IEEE Journal of Selected Topics in Applied Earth Observations And Remote Sensing, Vol. 7, No. 6, June 2014. 
[10] Suju Rajan, Joydeep 3 Ghosh, Fellow IEEE, and Melba M. Crawford, Fellow IEEE, “An Active Learning Approach to Hyperspectral Data Classification", IEEE Transactions on Geoscience and Remote Sensing, Vol. 46, No. 4, April 2008.

[11] Frederic Ratle, Gustavo Camps-Valls, Senior Member IEEE, and Jason Weston, "Semisupervised Neural Networks for Efficient Hyperspectral Image Classification", IEEE Transactions on Geoscience and Remote Sensing, Vol. 48. No. 5, pp. 2271 May 2010. 